\title{
Criticality in polar fluids
}

\author{
Yan Levin ${ }^{1,2 *}$, Paulo S. Kuhn ${ }^{1}$, Marcia C. Barbosa ${ }^{1}$ \\ ${ }^{1}$ Instituto de Física, Universidade Federal do Rio Grande do Sul \\ Caixa Postal 15051, CEP 91501-970, Porto Alegre, RS, Brazil \\ ${ }^{2}$ Institute for Theoretical Physics, University of California, \\ Santa Barbara, CA 93106-2431, USA \\ levin@if.ufrgs.br
}

(November 21, 2018)

\begin{abstract}
A model of polar fluid is studied theoretically. The interaction potential, in addition to dipoledipole term, possesses a dispersion contribution of the van der Waals-London form. It is found that when the dispersion force is comparable to dipole-dipole interaction, the fluid separates into coexisting liquid and gas phases. The calculated critical parameters are in excellent agreement with Monte Carlo simulations. When the strength of dispersion attraction is bellow critical, no phase separation is found.
\end{abstract}

PACS numbers: 61.20.Gy; 64.70.Fx; 75.50.Mm;

The phase behavior of simple molecular fluids was thought to be well understood since the early work of van der Waals over a century ago. It came, therefore, as a shock when the simulations in the early $90^{\prime} s$ could not locate the anticipated liquid-gas coexistence in the simplest model of polar fluid, idealized by dipolar hard spheres $D H S$ e 3 . The shock was further augmented by the fact that our everyday experience with polar fluids, such as water, does not leave any doubt that they do posses both liquid and gas phases. Furthermore, the theoretical methods such as,

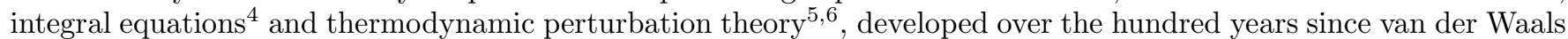
first proposed his celebrated theory, all predicted such a phase separation. What could have gone wrong?

The simulations of particles which interact by long ranged potentials, such as Coulomb or dipolar, are notorious for their difficulty. Thus, it is always possible that for some technical reason the simulations could not access the region of instability. This, however, seems to be less and less likely in view of constantly improving computational power and algorithm design. The first hint of what was happening was provided by the original simulations of Weis, Levesque, and Cailloll. These authors observed that at low temperatures, where the theories predicted phase separation into coexisting liquid and gas phases, the dipolar fluid became highlystructured, with particles forming chains of aligned dipoles. A further clue was obtained by van Leeuwen and Smit 2 who simulated soft dipolar particles in which the softcore repulsion, $1 / r^{12}$, was augmented by a variable isotropic attraction of van der Waals-London form $7,1 / r^{6}$. By performing the Gibbs-ensemble Monte Carlo simulation van Leeuven and Smit came to the conclusion that a minimum strength of isotropic attraction was necessary in order to produce phase separation. The ubiquity of liquidgas coexistence in real world can, therefore, be attributed to the presence of dispersion interactions between the molecules of polar fluids A number of theories have been put forward to try to explain the unusual behavior found in computer simulations $\mathrm{B}$ ed, but none has been able to completely resplve the mystery. Recently a new theory based on the pioneering ideas of Depye, Hücke11, Bjerrum12, and Onsager 13 has been advanced to account for the absence of phase separation in $\mathrm{DHS}_{\mathrm{S}} \mathrm{L}$. A similar approach has proyen to be successful in the study of coulombic criticality in restricted primitive model $(R P M)$ of electrolyte solutions 15 , predicting a coexistence curve in good agreement with experiments and computer simulations 16 . From the theoretical perspective, however, the system simulated by van Leeuven and Smitl is significantly more complex than the RPM or the DHS. Besides the dipole-dipole interaction the theory must be able to deal with the softcore repulsion, as well as with the short ranged dispersion attraction. This model, therefore, provides a very stringent test of any theory of criticality in polar fluids. A successful theory should account for both, the presence of phase separation for strong dispersion forces, as well as for its disappearance when the isotropic coupling is reduced bellow the critical value.

Our model, then, consists of $N$ molecules of dipole moment $\boldsymbol{p}$, inside a uniform medium of dielectric constant $\epsilon_{0}$. The interaction potential between any two particles is

$$
U\left(\boldsymbol{r} ; \boldsymbol{p}_{1}, \boldsymbol{p}_{2}\right)=\frac{p^{2}}{\epsilon_{0} \sigma^{3}}\left(\left(\frac{\sigma}{r}\right)^{12}-\lambda\left(\frac{\sigma}{r}\right)^{6}\right)+\frac{1}{\epsilon_{0} r^{3}}\left(\boldsymbol{p}_{1} \cdot \boldsymbol{p}_{2}-\frac{3\left(\boldsymbol{p}_{1} \cdot \boldsymbol{r}\right)\left(\boldsymbol{p}_{2} \cdot \boldsymbol{r}\right)}{r^{2}}\right) .
$$

The dimensionless parameter $\lambda$ defines the strength of the isotropic interaction as it compares with dipole-dipole electrostatic potential. To proceed we associate with the model of "soft" molecules given by Eq. (11) an equivalent

${ }^{*}$ Corresponding author 
system of "rigid" particles. The advantage of working with hard particles is that a number of analytic equations of state have been developed to study rigid molecules, in particular hard spheres. Fixing one molecule at the origin with the dipole moment aligned with the z-axis and a second particle at $\boldsymbol{r}$ with the dipolar orientation $\boldsymbol{\Omega}$, the potential in Eq. (11) can be separated in two parts 2 . The repulsive term, $U_{r p}(\boldsymbol{r}, \boldsymbol{\Omega})=U\left(\boldsymbol{r} ; \boldsymbol{p}_{1}, \boldsymbol{p}_{2}\right)-U\left(\boldsymbol{r}_{\min }, \boldsymbol{\Omega}_{\min }\right)$ for $|\boldsymbol{r}|<\left|\boldsymbol{r}_{\text {min }}\right|$ and $U_{r p}(\boldsymbol{r}, \boldsymbol{\Omega})=0$ for $|\boldsymbol{r}| \geq\left|\boldsymbol{r}_{\text {min }}\right|$; and the attractive term, $U_{a t}(\boldsymbol{r}, \boldsymbol{\Omega})=U\left(\boldsymbol{r}_{\text {min }}, \boldsymbol{\Omega}_{\text {min }}\right)$ for $|\boldsymbol{r}|<\left|\boldsymbol{r}_{\text {min }}\right|$ and $U_{a t}(\boldsymbol{r}, \boldsymbol{\Omega})=U\left(\boldsymbol{r} ; \boldsymbol{p}_{1}, \boldsymbol{p}_{2}\right)$ for $|\boldsymbol{r}| \geq\left|\boldsymbol{r}_{\min }\right|$. Here $\boldsymbol{r}_{\text {min }}$ is the intermolecular vector and $\boldsymbol{\Omega}_{\min }$ is the dipolar orientation for which the interaction potential is minimum. In the spirit of Weeks, Chandler, and Andersen (WCA) $t 7$ we can associate with the soft particles given by Eq. (1) a system of rigid dipoles interacting by

$$
\begin{aligned}
& U^{d d}(\boldsymbol{r})=-\frac{\lambda p^{2} a^{3}(\theta ; \lambda)}{\epsilon_{0} r^{6}}+\frac{1}{\epsilon_{0} r^{3}}\left(\boldsymbol{p}_{1} \cdot \boldsymbol{p}_{2}-\frac{3\left(\boldsymbol{p}_{1} \cdot \boldsymbol{r}\right)\left(\boldsymbol{p}_{2} \cdot \boldsymbol{r}\right)}{r^{2}}\right) \boldsymbol{r} \geq|\mathbf{a}| \\
& U^{d d}(\boldsymbol{r})=\infty \boldsymbol{r}<|\mathbf{a}|
\end{aligned}
$$

where the distance of closest approach $a(\theta ; \lambda)$ is determined by the condition, $U_{r p}\left(\mathbf{a}, \boldsymbol{\Omega}_{\min }\right)=k_{B} T$. In view of the azimuthal symmetry, $|\mathbf{a}|=a(\theta ; \lambda)$ is only a function of the angle $\theta$ between the dipole moment $\boldsymbol{p}_{1}$ and the intermolecular vector $\boldsymbol{r}$. To stress that this distance depends on the strength of the isotropic attraction, we have included $\lambda$ as an explicit parameter in $a(\theta ; \lambda)$. The excluded volume region around the central dipole has the form of geoid, a flattened sphere, with the eccentricity a decreasing function of $\lambda$ (the highest eccentricity for $\lambda=0$ ).

The reduced free energy density, $f=\beta F / V$, of a fluid with the interaction potential Eq. 2 can be constructed as a sum of terms embodying the most relevant physical features, starting with the entropic ideal gas contribution, $f^{i d}=\rho \ln \left(\rho \Lambda^{3}\right)-\rho$. Here $\rho=N / V$ is the density of dipoles, $\beta=1 / k_{B} T$, and $\Lambda$ is the thermal wavelength. The mean energy of interaction between the dipoles can be calculated as

$$
F^{v d W}=\frac{1}{2} \int d \mathbf{r}_{1} d \mathbf{r}_{2} \rho\left(\mathbf{r}_{1}\right) \rho\left(\mathbf{r}_{2}\right) U^{d d}\left(\mathbf{r}_{1}, \mathbf{r}_{2}\right),
$$

where $\rho(\mathbf{r})$ is the local density of particles and where, to simplify the notation, we have suppressed integration over the relative orientation of dipole moments. Since the distribution of particles and their orientation is uniform, only the first term of Eq. (2) contributes to the integral, with the second term averaging to zero. In order to simplify the integration we can replace the excluded volume region by a sphere with radius $a_{s}(\lambda)=(a(0 ; \lambda)+2 a(\pi / 2 ; \lambda)) / 3$. Since the eccentricity is quite small this is a good approximation. We have numerically checked that the error introduced by this approximation is no more than a few percent. Performing the integration, we find the reduced free energy due to dispersion interactions to be

$$
f^{v d W}=-\frac{2 \pi}{3 a_{s}^{3}(\lambda)} \frac{\lambda\left(\rho^{*}\right)^{2}}{T^{*}}
$$

where we have defined the reduced (dimensionless) temperature and density to be, $T^{*}=k_{B} T \epsilon_{0} \sigma^{3} / p^{2}$ and $\rho^{*}=\rho \sigma^{3}$, respectively.

Although on average the dipoles are distributed uniformly, with no particular preference for any specific orientation, the long ranged dipolar force produces strong correlations between the dipole moments of individual particles. This leads to a significant contribution to the total free energy. To obtain this correlational free energy, we fix one particle at the origin with its dipole moment aligned with the z-axis. The electrostatic potential that this dipole feels due to the presence of other molecules can be found from the solution of Laplace equation $\nabla^{2} \phi=0$. The boundary conditions require continuity of the potential, $\phi_{\text {in }}\left(a_{s}\right)=\phi_{\text {out }}\left(a_{s}\right)$, and the displacement field, $\epsilon_{0} \phi_{\text {in }}^{\prime}\left(a_{s}\right)=\epsilon \phi_{\text {out }}^{\prime}\left(a_{s}\right)$, across the surface $r=a_{s}$. The susceptibility of the outer dipoles to polarization by the electric field can be characterized by the repgrmalized dielectric constant $\epsilon$, the expression for which can be obtained from the Onsager's reaction field theory 13 , $\left(\epsilon-\epsilon_{0}\right)\left(\epsilon_{0}+2 \epsilon\right) / \epsilon=4 \pi \beta p^{2} \rho$. The Laplace equation can now be integrated to yield the electric field felt by the central dipole due to the other particles,

$$
\mathbf{E}_{0}=\frac{2 p}{\epsilon_{0} a_{s}^{3}(\lambda)} \frac{\epsilon-\epsilon_{0}}{2 \epsilon+\epsilon_{0}} \hat{\mathbf{z}}
$$

The electrostatic free energy of the whole system is obtained from the Debye charging process 1 . 15 in which all the particles are charged simultaneously from zerg to their final dipolar strength. The integration can be done explicitly yielding the correlational free energy density 1422 ,

$$
f^{d d}=-\frac{1}{4 \pi a_{s}^{3}(\lambda)}\left\{-2+\frac{1}{\psi(u)}+\psi(u)+\frac{9}{2} \ln \left(\frac{3}{2 \psi(u)+1}\right)+3 \ln \psi(u)\right\}
$$


with $\psi(u) \equiv \epsilon(u) / \epsilon_{0}=\frac{1}{4}(1+u)+\frac{1}{4} \sqrt{9+2 u+u^{2}}$, and $u=4 \pi \rho^{*} / T^{*}$. Finally, the contribution to the total free energy due to hardcore repulsion can be approximated by the Carnahan-Starling $(C S)$ form $\mathbb{2}^{3}, f^{h c}=\rho g(\eta)$, where $g(\eta)=\eta(4-3 \eta) /(1-\eta)^{2}$ and $\eta=\pi a_{s}^{3}(\lambda) \rho / 6$ is the volume fraction occupied by dipoles.

Combining all of the contributions, the total free energy of dipolar fluid becomes $f=f^{i d}+f^{h c}+f^{v d W}+f^{d d}$. It is a simple matter to see that for any $\lambda$ this free energy violates the convexity requirement when the temperature is lowered below the critical value $T_{c}(\lambda)$. The critical parameters are plotted in Fig. 1 . It is evident that the agreement with the simulations is quite good. For $0.3<\lambda \leq 1$, the critical densities are close to the ones obtained in the simulations.
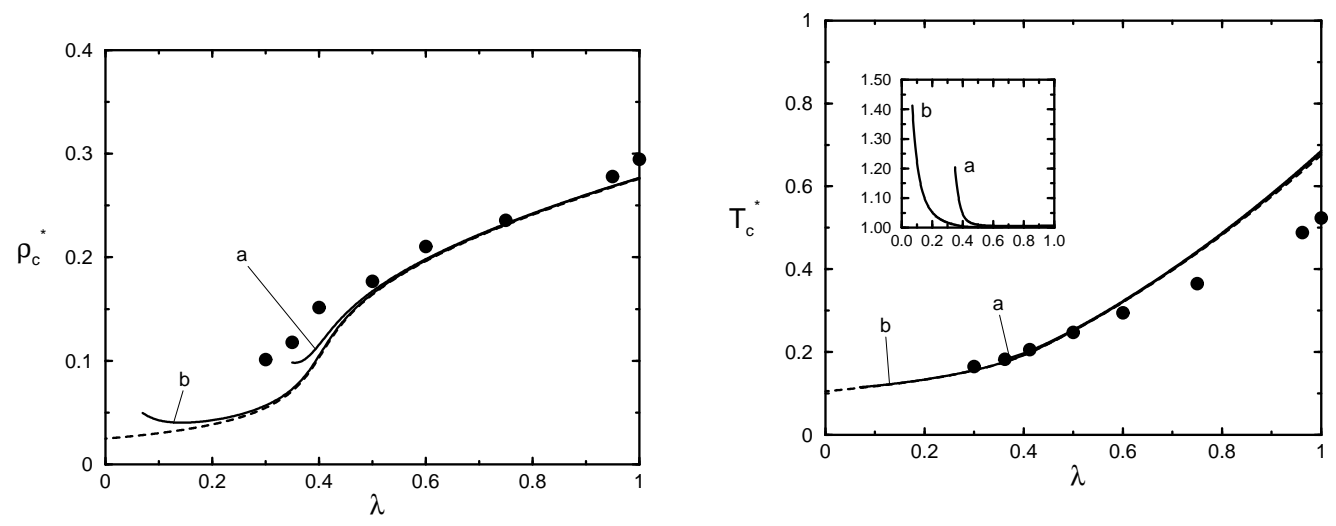

FIG. 1. Critical density and critical temperature as a function of $\lambda$ : the dashed curve is for the theory without association; the two solid curves $a$ and $b$ are for the limiting cases $h(n) \approx 1.52165 \ldots$ and $h(n) \approx 1.19055 \ldots$, respectively. Solid circles are the Monte Carlo data from Ref[] $]$. Inset shows the average cluster size as a function of $\lambda$.

For $\lambda=1$ the critical temperature is about $30 \%$ too high. However, for smaller isotropic couplings, $\lambda<0.6$, this discrepancy almost completely disappears. It is not surprising that for $\lambda \approx 1$ the agreement is the poorest. After all, the theory presented above is intrinsically mean-field, and as such neglects the thermal fluctuations which are the strongest in the vicinity of the critical point. For short ranged potentials, the effect of fluctuations is to significantly depress the criticaltemperature. Even for such relatively simple system as "argon" mean-field theories overestimate $T_{c}$ by $15 \%$ to $20 \%$. We might expect that as the relative strength of isotropic short ranged interaction decreases, compared to the long ranged dipolar force, the effect of fluctuations on the critical temperature will also diminish. This, indeed, is the case, see Fig. 1 . We note that for $\lambda \approx 1$ the phase separation is the result of the competition between the attractive and the excluded volume, $C S$, interactions. For small $\lambda^{\prime} s$, on the other hand, the instability is purely electrostatic and is only weakly affected by the hardcore contribution to the total free energy.

For $\lambda<0.3$ the simulations fail to find any coexistence, while our theory predicts phase separation all the way down to $\lambda=0$. What is responsible for this discrepancy? In view of the previous discussion, we do not have to look too far for an explanation. It was observed in simulations that for $\lambda<0.3$, the low temperature thermodynamics was dominated by polymer-like chains of aligned dipoles. On the other hand, the theory presented above is intrinsically linear, and does not take into account the strong non-linear effects associated with the formation of clusters. To account for these, while preserving the theory's linear structure, we shall explicitly allow for chains composed of $n$ associated dipoles. Based on the Monte Carlo simulations we shall ignore other possible geometries of chisters. This, actually, is a non-trivial approximation, since at zero temperature the energy favors compact clusters 14 . The simulations, however, find that for finite temperatures linear chains predominate. Thus, as a first approximation we shall only consider polymer-like clusters.

The density of chains containing $n$ monomers will be denoted by $\rho_{n}$, with the density of free, unassociated, dipoles $\rho_{1}$. The particle conservation leads to $\rho=\sum_{n=1}^{\infty} n \rho_{n}$, where the total density of dipoles is $\rho=N / V$. This is the so called "chemical", as opposed to "physical" picture of statistical mechanics of strongly interacting particles. It is important to keep in mind that no new approximations are being introduced into the theory, but only a change of perspective. Clearly if, as it happens at high temperatures, the formation of clusters is unlikely, the theory should inform us of this fact by predicting low cluster density. As was noted by Onsager "what we remove from one page of the ledger would be entered elsewhere with the same effect" 19 . In fact it is possible to directly map this chemical picture onto exact thermodynamic perturbation theory 20 . The new perspective is especially useful at low temperatures, where the perturbation theory is very difficult to construct, but where the "chemical" view point provides a particularly clear perspective of the physical phenomena. We can now proceed to make the first approximation. In the spirit of Bjerrum12, we shall assume that the clusters interact only weakly with each other and with the free dipoles. Thus, as 
a second approximation, we shall treat clusters as ideal non-interacting species with all of the interactions confined to free dipoles. This approximation has proven to work quite well in the theory of electrolytes, in particular allowing for a precise location of the critical point 15 .

The entropic free energy due to thermal motion of dipoles and clusters can be approximated by the ideal gas contribution, $f^{i d}=\sum_{n=1}^{\infty} \rho_{n} \ln \left(\rho_{n} \Lambda^{3 n} / \zeta_{n}\right)-\rho_{n}$. In the limit of low temperatures, the internal partition function of an $n$ cluster, $\zeta_{n}$, can be evaluated for chainlike configurations to yield 14.21

$$
\zeta_{n}=\left\{\frac{\pi^{3 / 2} 2^{21 / 18} T^{* 5 / 2} \sigma^{3}}{9 \sqrt{3}}\right\}^{n-1} \exp \left\{\frac{(n-1) h(n)}{T^{*}}\right\}
$$

where,

$$
h(n)=3\left[\frac{n\left[\psi^{(2)}(n)-\psi^{(2)}(1)\right]+2\left[\psi^{(1)}(n)-\psi^{(1)}(1)\right]}{4(n-1)}\right]^{4 / 3} .
$$

The $\psi^{(1)}(n)$ and $\psi^{(2)}(n)$ are the polygamma functions of the first and second order, respectively. For free dipoles $\zeta_{1}=1$. The hardcore contribution can, once again, be approximated by the Carnahan-Starling form, $f^{h c}=\sum_{n=1}^{\infty} \rho_{n} g(\eta)$, where $\eta=\pi a_{s}^{3}(\lambda) \rho / 6$ is the total volume fraction occupied by dipoles and chains. The mean-field and the electrostatic contributions to the free energy are given by Eqns. (4i) and (6), with the total density $\rho$ replaced by the density of free dipoles $\rho_{1}$. The distribution of cluster densities can be obtained through minimization of the total free energy $f=f^{i d}+f^{h c}+f^{v d W}+f^{d d}$, subject to constraint of particle conservation. This reduces to the law of mass action, $\mu_{n}=n \mu_{1}$, relating the chemical potential of free dipoles $\mu_{1}=\partial f / \partial \rho_{1}$, to the chemical potential of $\mathrm{n}$ clusters $\mu_{n}=\partial f / \partial \rho_{n}$. Substituting the free energy, the low of mass action yields

$$
\rho_{n}=\zeta_{n} \rho_{1}^{n} e^{\beta n \mu^{e x}+(n-1) g(\eta)}, n \geq 2 ;
$$

where the excess chemical potential is $\mu^{e x}=\partial f^{d d} / \partial \rho_{1}$. In spite of its simple appearance the law of mass action is actually an infinite set of coupled algebraic equations which determine the distribution of chain densities $\left\{\rho_{n}\right\}$. To solve these equations is a difficult numerical task. In order to proceed we observe that $h(n)$ is a uniformly increasing function bounded by the two limiting values, $h(2)=1.19055 \ldots$ and $h(\infty)=1.52165 \ldots ;$ i.e. $1.19055 \ldots \leq h(n) \leq 1.52165 \ldots \forall n$. Use of these bounds allows us to delimitate the critical parameters. Approximating $h(n)$ by one of the limiting values, Eq. (9) can be summed explicitly, $\sum_{n=2}^{\infty} n \rho_{n}=\rho-\rho_{1}$, reducing to a single algebraic equation for $\rho_{1}$. Substituting the root of this equation back into Eq. (9) leads to the distribution of cluster sizes. For large $\lambda^{\prime} s$, separation in two coexisting phases is once again found. In fact, the critical densities and temperatures are identical to our earlier calculation done in the absence of cluster formation. As $\lambda$ decreases, the critical temperature also diminishes, and dipolar association becomes relevant. We find that below certain critical value, $0.07<\lambda_{c}<0.35$, the phase separation disappears, see Fig. 1. Van Leeuwen and Smit could not locate phase separation for $\lambda<0.3$, the value which is very close to our upper bound. We note, however, that the lower bound is probably more realistic since, as can be seen from the inset to Fig 1, the average chain size is still quite small in the region of criticality.

We conclude that the theory presented above captures the essential physics of criticality in polar fluids. A number of issues still need to be addressed. As it stands, the theory does not take into account interactions between the chains. How these residual forces can be introduced into the theory is far from obvious. We must stress, however, that the naive approach of allgwing each one of the monomers inside the chains to interact with unrenormalized potential, Eq. (1), is incorrect24. This would double count the interactions, which are partially taken care of by the dipolar association, and would lead to phase separation for all values of $\lambda$.

The nature of criticality in polar fluids is also of great interest. Although there can be little doubt that the Stockmayer fluid $(\lambda=1)$ belongs to the Ising universality class, in view of our results, this no longer seems to be so evident for dipolar fluids with $\lambda \approx 0.3$. The fact that the mean-field theory can account so well for the location of the critical point, suggests that for $\lambda<0.5$ the fluctuations play only a marginal role. One might, therefore, expect to see mean-field critical exponents, or a crossover from the mean-field to the Ising universality in a very narrow neighborhood of the critical point. As is the case for electrolytes, the criticality in dipolar systems should remain a challenge for the foreseeable future.

While this paper was going through the refereeing process, new simulations 25 have once again raised a possibility of the phase separation of $D H S$ at sufficiently low temperatures and densities. Unlike, the usual gas-liquid transition, it has been argued, however, that this novel phase separation is driven by the proliferation of Y-like bifurcations26. The two coexisting phases are found to have respectively a large and a small concentration of Y-like defects 27 .

This work was supported in part by Conselho Nacional de Desenvolvimento Científico e Tecnológico (CNPq), Financiadora de Estudos e Projetos (FINEP), and by the National Science Foundation (NSF) under Grant No. PHY94-07194. 
${ }^{1}$ J.J. Weis and D. Levesque, Phys. Rev. Lett. 71, 2729, (1993); J.-M. Caillol, J. Chem. Phys, 98, 9835, (1993).

${ }^{2}$ M.E. van Leeuwen and B. Smit, Phys. Rev. Lett. 71, 3991, (1993).

${ }^{3}$ M.J. Stevens and G.S. Grest, Phys. Rev. Lett. 72, 3686, (1994).

${ }^{4}$ M.S. Wertheim, J. Chem. Phys. 55, 4291, (1971).

${ }^{5}$ P.G. de Gennes and P.A. Pincus, Phys. Kondens. Mater. 11, 189 (1970).

${ }^{6}$ G.S. Rushbrooke, G. Stell, and J.S. Høye, Molec. Phys. 26, 1199, (1973).

${ }^{7}$ See also B. Groh and S. Dietrich, Phys. Rev. E 50, 3814 (1994); Phys. Rev. E 54, 1687 (1996); Phys. Rev. Lett. 79, 749 (1997).

${ }^{8}$ R. P. Sear, Phys. Rev. Lett. 76, 2310, (1996).

${ }^{9}$ R. van Roij, Phys. Rev. Lett. 76, 3348, (1996).

10 J.M. Tavares, M.M. Telo da Gama, and M. A. Osipov, Phys. Rev. E 56, R6252, (1997); Phys. Rev. E 577367 (E) (1998); M.A. Osipov, P.I.C. Teixeira, M.M. Telo da Gama, Phys. Rev. E 54, 2597, (1996).

${ }^{11}$ P.W. Debye and E. Hückel, Phys. Z. 24, 185, (1923).

12 N. Bjerrum, Kgl. Dan. Vidensk. Selsk. Mat.-fys. Medd. 7, 1, (1926).

${ }^{13}$ L. Onsager, J. Am. Chem. Soc. 58, 1486, (1936).

${ }^{14}$ Y. Levin, Phys. Rev. Lett. 83, 1159, (1999).

${ }^{15}$ M.E. Fisher and Y. Levin, Phys. Rev. Lett. 71, 3826, (1993); Y. Levin and M.E. Fisher, Physica A 255,164 (1996).

${ }^{16}$ J.P. Valleau, J. Chem. Phys. 95, 584, (1991); A.Z. Panagiotopoulos, Fluid Phase Equib. 76, 92, (1993).

17 J.D. Weeks, D. Chandler, H.C. Andersen, J. Chem. Phys. 54, 5237, (1971); D. Chandler, J.D. Weeks, H.C. Andersen, Science 220, 787, (1983).

${ }^{18}$ M.E. Fisher, J. Stat. Phys. 75, 1, (1994);

${ }^{19}$ H. Falkenhagen, W. Ebeling, in: Ionic Interactions, ed. S. Petrucci (Academic Press, NY, 1971) Vol. 1.

${ }^{20}$ T.L. Hill, Statistical Mechanics: Principles and Selected Applications, (McGraw-Hill, NY, 1956).

${ }^{21}$ P.C. Jordan, Molec. Phys. 25, 961, (1973).

${ }^{22}$ see also G. Nienhuis and J. M. Deutch, J. Chem. Phys. 55, 4213, (1971); J. W. Sutherland, G. Nienhuis, and J.M. Deutch, Mol. Phys. 27, 721, (1974); V.C. Weiss and W. Schröer, J. Chem. Phys. 108, 7747, (1998).

${ }^{23}$ N.F. Carnahan, K.E. Starling, J. Chem. Phys. 51, 635, (1969).

${ }^{24}$ M.E. Fisher, D.M. Zuckerman, J. Chem. Phys. 109, 7961, (1998).

${ }^{25}$ P. J. Camp, J.C. Shelley, and G.N. Patey, Phys. Rev. Lett. 84, 115, (2000).

${ }^{26}$ T. Tlusty and S.A. Safran, Science 290, 1328, (2000).

${ }^{27}$ P. Pincus, Science 290, 1307, (2000). 